\title{
Prompt admission to the ICU : an instrument to improve mortality for deteriorating ward patients
}

\section{Lange, Theis}

2018-05

Lange , T , Skrifvars , M \& Ranzani , O T 2018 , ' Prompt admission to the ICU : an instrument to improve mortality for deteriorating ward patients ', Intensive Care Medicine , vol. 44 , no. 5 , pp. 678-680 . https://doi.org/10.1007/s00134-018-5194-9

http://hdl.handle.net/10138/237104

https://doi.org/10.1007/s00134-018-5194-9

unspecified

publishedVersion

Downloaded from Helda, University of Helsinki institutional repository.

This is an electronic reprint of the original article.

This reprint may differ from the original in pagination and typographic detail.

Please cite the original version. 


\section{EDITORIAL}

\section{Prompt admission to the ICU: an instrument to improve mortality for deteriorating ward patients}

Theis Lange ${ }^{1,2^{*}}$, Markus Skrifvars ${ }^{3,4}$ and Otavio T. Ranzani ${ }^{5}$

๑ 2018 Springer-Verlag GmbH Germany, part of Springer Nature and ESICM

\section{Introduction}

In a recent article in Intensive Care Medicine, Harris and co-authors present a study on the importance of prompt transfer of deteriorating ward patients to the ICU [1]. Between 2010 and 2011, they conducted a large observational study involving 49 hospitals in the UK considering the problem of ongoing bed block causing unwanted delays in the admission of patients. We would like to congratulate the authors on conducting such challenging and groundbreaking study designed to answer a valid but also somewhat delicate research question. A key feature of this study is the applied instrumental variable (IV) analysis, which we believe has a non-harvested potential within critical care research.

\section{Do the efforts for a fast ICU transfer make a difference?}

The assessment of deteriorating ward patients is a key part of the daily work for every intensivist. This involves many complicated questions: "Will this patient benefit from ICU admission?", or "How many free beds do we have in the ICU now and later?". Indeed, triage decisions are embedded in the complex environment of each hospital, where the ICU capacity and the efficiency on admitting or discharging patients without delays are crucial [1-3]. When the decision for an ICU transfer is made, the ward, rapid response and ICU teams usually feel relieved when the process is fast. However, in real

\footnotetext{
*Correspondence: thlan@sund.ku.dk

1 Section of Biostatistics, University of Copenhagen, Copenhagen, Denmark

Full author information is available at the end of the article
}

life, there are multiple (expected and unexpected) bottlenecks that need to be overcome [4-6].

To randomize to prompt or "watchful waiting" ICU admission for deteriorating ward patients is likely to lack clinical equipoise and therefore difficult to study with a randomized design [7]. Thus, Harris and co-authors settled on an intelligent cohort study, starting from ward patients referred for assessment by critical care teams. They followed these patients prospectively, including those recommended and those not recommended for ICU admission, and were able to capture outcomes for reviewed patients by linking data specifically collected in this study to the Intensive Care National Audit \& Research Centre (ICNARC) database.

In the analysis, the authors applied IV methodology to estimate the causal effect of prompt transfers $(<4 \mathrm{~h}$ of assessment) compared to what they defined as a "watchful waiting" cohort. The authors observed that prompt admissions reduced 90-day mortality in the whole cohort by $7.4 \%$ (95\% CI $1.7,18.5 \%)$ and by $16.2 \%$ (95\% CI $1.1,31.3 \%$ ) in those recommended for immediate ICU admission.

Although the study and the IV method have some limitations as discussed in the manuscript, the (SPOT)light results highlight the need for optimization of the patient's flow and ICU efficiency. The authors observed that transfer delays are frequent, related to ICU strain and associated with worse outcome. The solutions are not easy and require efforts from several players within the hospital. The current study, especially if replicated in other settings, highlights the need for more research on ICU triage and discharge decisions. Solutions could include incorporating concepts from other fields, such as engineering and logistics [8]. 


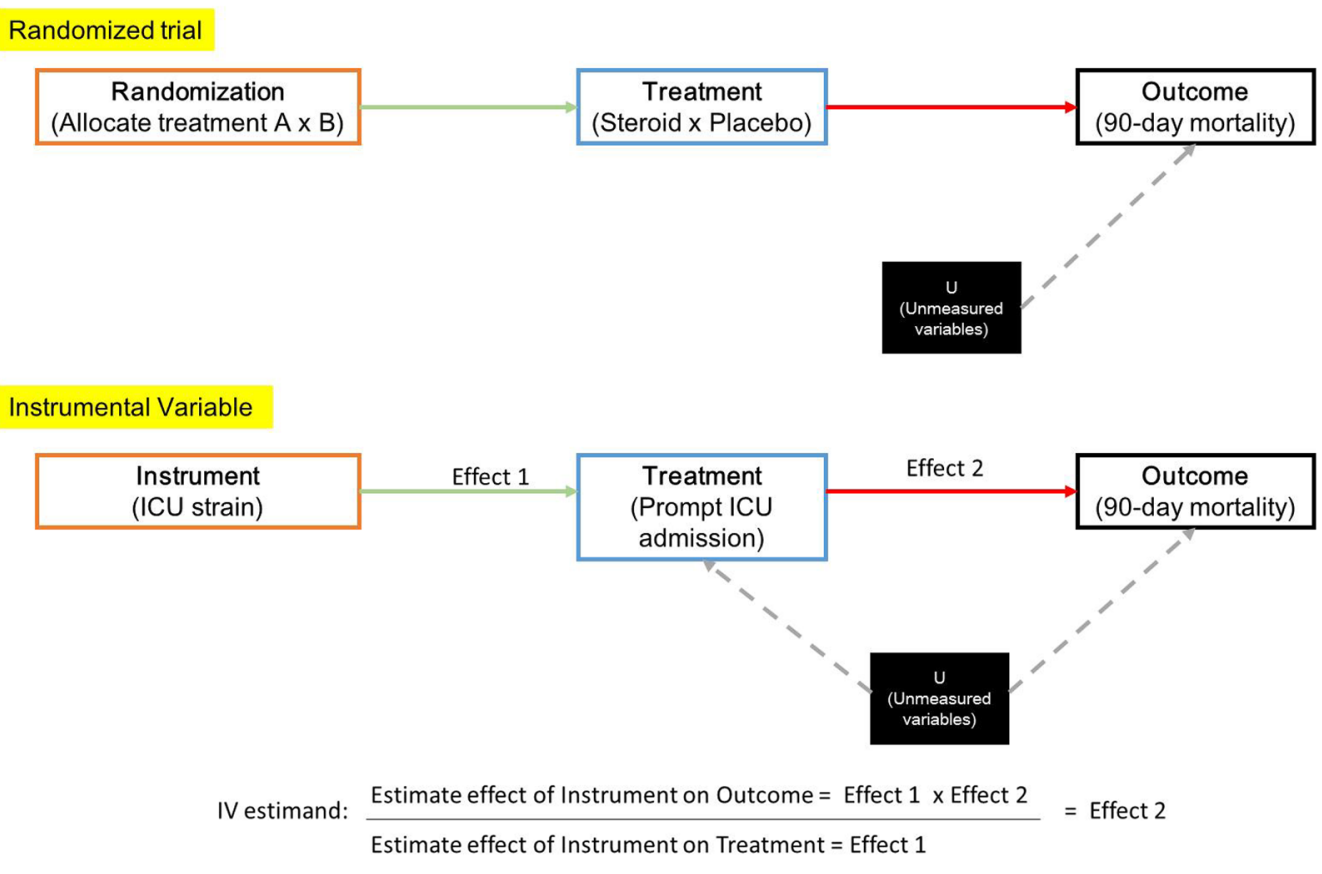

Fig. 1 Causal directed acyclic graphs illustrating a randomized controlled trial and an estimation based on instrumental variable analysis

\section{IV method: what it is and which potentials?}

A randomized controlled trial (RCT) is the gold standard for drawing a causal conclusion because its design ensures that treatment allocation does not depend on any known or unknown patient characteristics. In contrast, observational studies do not have randomization to ensure treatment groups are comparable, increasing the possibility of confounding. Accordingly, an attempt is made to collect information on as many potential confounders as possible with adjustment for imbalances using statistical methods. However, it will always be a matter of faith if we trust that enough confounders have been collected. Presented like this, it appears that the two study designs are completely distinct, but the truth is that they are just in the opposite ends of a continuum of study designs where we have more or less control of how treatment is allocated $(100 \%$ control in RCT; $0 \%$ in the other end). Re-examining the RCT reveals that the important part is not that we have $100 \%$ control of the treatment allocation mechanism, but instead it is that we by design introduce a component in the treatment allocation which is unrelated to characteristics of the included patient. This is sometimes called an exogenous push (i.e. the instrument).

The key idea in IV analyses is that this exogenous push could also come from sources other than randomization.
Harris et al. use the fact that overall bed occupancy in the ICU (the instrument) will affect the probability of prompt admission to ICU irrespective of the specific clinical condition of that patient. Or phrased differently: there are patients who had they been presented on a day with many open beds in the ICU would have been given prompt admission, but had the same patients been presented on a day with few free beds in the ICU they would not be given prompt admission. The link between randomization and IV appear clear in this setting. Methods for handling non-compliance in RCTs [9-11] are also a case of IV analyses.

The precise details on IV estimation depend on outcome and instrument type; see Greenland [12] for an introduction, but the basic idea is always the same: (1) estimate the causal effect of the instrument on the outcome. This effect can be thought of as the multiplication of the causal effect of the instrument on the treatment and the causal effect of the treatment on outcome. (2) Estimate the causal effect of the instrument on the exposure. (3) Divide the estimate in one by the one from two to produce an estimate of the causal effect of treatment on exposure. The method only works under the (untestable) assumption that the IV only affects the considered outcomes through its effect on treatment. With bed occupancy as the instrument, this assumption is 
not trivial as one could fear that treatment quality in the ICU is reduced in conditions of high bed occupancy; this potential problem is, however, addressed in the paper. No knowledge of confounding factors between prompt admission and survival is required. We only need to know that there are no confounding factors between instrument and exposure and instrument and outcome; see Fig. 1 for a graphical illustration of the assumptions.

The statistical methods for conducting IV analysis are still developing (see e.g. Tchetgen Tchetgen [13] for survival outcomes) and they are a bit harder to implement than standard regression. However. these are just technicalities which should not distract us from the essential point: the analysis by Harris illustrates how IV methods can replace the need for detailed clinical information on each patient with simple administrative data on hospital utilization. The latter data is much "easier" to come by than the first (without in anyway belittling the work done by the authors). The new requirement is that researchers must think outside the usual suspects of variables influencing treatment allocation. We believe that such variables are common (e.g. occupancy, administrative changes, changes in funding of wards, changes in uptake areas, etc.) and we encourage our colleagues to "go look for that exogenous pushing".

\section{Author details \\ ${ }^{1}$ Section of Biostatistics, University of Copenhagen, Copenhagen, Denmark. ${ }^{2}$ Center for Statistical Science, Peking University, Beijing, China. ${ }^{3}$ Department of Anaesthesiology, Intensive Care and Pain Medicine, University of Helsinki and Helsinki University Hospital, Helsinki, Finland. ${ }^{4}$ Department of Emergency Medicine and Services, Helsinki University Hospital and University of Helsinki, Helsinki, Finland. ${ }^{5}$ Pulmonary Division, Heart Institute (InCor), Hospital das Clinicas HCFMUSP, Faculdade de Medicina, Universidade de Sao Paulo, São Paulo, Brazil.}

\section{Compliance with ethical standards}

\section{Conflicts of interest}

The authors declare that they have no conflicts of interest.
Received: 16 April 2018 Accepted: 18 April 2018

Published online: 28 April 2018

\section{References}

1. Harris Steve, Singer Mervyn, Sanderson Colin, Grieve Richard, Harrison David, Rowan Kathy (2018) Impact on mortality of prompt admission to critical care for deteriorating ward patients: an instrumental variable analysis using critical care bed strain. Intensive Care Med. https://doi. org/10.1007/s00134-018-5148-2

2. Sprung $C L$, Danis M, lapichino $G$ et al (2013) Triage of intensive care patients: identifying agreement and controversy. Intensive Care Med 39:1916-1924

3. Rhodes A, Ferdinande P, Flaatten H, Guidet B, Metnitz PG, Moreno RP (2012) The variability of critical care bed numbers in Europe. Intensive Care Med 38:1647-1653

4. Guidet B, Leblanc G, Simon T et al (2017) Effect of systematic intensive care unit triage on long-term mortality among critically III elderly patients in France. JAMA 318:1450

5. Ramos JGR, Perondi B, Dias RD et al (2016) Development of an algorithm to aid triage decisions for intensive care unit admission: a clinical vignette and retrospective cohort study. Critical Care. 20:81. https://doi. org/10.1186/s13054-016-1262-0

6. Bagshaw SM, Wang X, Zygun DA et al (2018) Association between strained capacity and mortality among patients admitted to intensive care: a path-analysis modeling strategy. J Crit Care 43:81-87

7. Stephens R, Cudnik M, Patterson E (2011) Barriers and facilitators to timely admission and transfer of patients from an emergency department to an intensive care unit. Proc Hum Factors Ergon Soc Annu Meet 55:763-767

8. Valley TS, Sjoding MW, Ryan AM, Iwashyna TJ, Cooke CR (2015) Association of intensive care unit admission with mortality among older patients with pneumonia. JAMA 314:1272

9. Kim S-H, Chan CW, Olivares M, Escobar GJ (2016) Association among ICU congestion, ICU admission decision, and patient outcomes* . Crit Care Med 44:1814-1821

10. Li Bassi G, Panigada M et al (2017) Randomized, multicenter trial of lateral Trendelenburg versus semirecumbent body position for the prevention of ventilator-associated pneumonia. Intensive Care Med 43:1572-1584

11. Sussman JB, Hayward RA (2010) An IV for the RCT: using instrumental variables to adjust for treatment contamination in randomised controlled trials. BMJ 340:c2073

12. Greenland S (2008) An Introduction to instrumental variables for epidemiologists. Int J Epidemiol 29:722-729

13. Tchetgen Tchetgen EJ, Walter S, Vansteelandt S, Martinussen T, Glymour M (2015) Instrumental variable estimation in a survival context. Epidemiology 26:402-410 